\title{
Low Grazing Incidence Millimeter-Wave Scattering Models and Measurements for Various Road Surfaces
}

\author{
Eric S. Li, Member, IEEE, and Kamal Sarabandi, Senior Member, IEEE
}

\begin{abstract}
Systematic characterization of the scattering behavior of traffic targets, clutter, and their associated interactions are required in order to design and assess the performance of millimeter-wave-based sensors for automated highway system (AHS) applications. In this paper, the polarimetric radar backscatter response of various road surfaces is investigated both theoretically and experimentally. In general, it is found that the overall scattering response of road surfaces is composed of volume and surface scattering components. Recently a hybrid volume scattering model was developed for predicting the backscatter response of smooth asphalt surfaces at millimeterwave frequencies. There, only the volume scattering was accounted for, however, experimental results show that the surface scattering cannot be ignored when the surface roughness parameters become comparable to the radar wavelength. In this paper, the previous study is extended to include the radar backscatter response of concrete surfaces, snow-covered smooth surfaces, and rough asphalt or concrete surfaces. Radiative transfer (RT) theory is used to model the volume scattering and the integral equation model is used to describe the surface scattering. Asphalt and concrete mixtures are dense random media whose extinction and phase matrices are characterized experimentally. Ice and water over asphalt and concrete surfaces are modeled by homogeneous layers. Fresh snow is modeled by a sparse random medium whose extinction and phase matrices are obtained analytically. The University of Michigan 94-GHz polarimetric radar system was used to perform polarimetric backscatter measurements of the aforementioned road surfaces at near grazing incidence angles $\left(70^{\circ}-88^{\circ}\right)$. Comparison of the measured and theoretically predicted backscattering coefficients and polarimetric phase difference statistics shows excellent agreement.
\end{abstract}

Index Terms - Electromagnetic scattering, millimeter wave, road surfaces.

\section{INTRODUCTION}

I $\mathrm{N}$ response to the increasing demand for safety in highway transportation, different types of radar-based sensors have been proposed. These include forward looking millimeterwave radars for collision warning and intelligent cruise control, surface recognition sensor, lane change aid radar, etc. Design of optimum systems requires a thorough understanding of the radar phenomenology of targets in the highway environment. This includes road surfaces where their radar response is important in the design of road surface recognition sensors, and in characterization of signal-to-clutter ratio for obstacle detection

Manuscript received April 28, 1998; revised February 15, 1999. This work was supported by the National Automotive Highway Systems Consortium (NAHSC).

E. S. Li is with EMAG Technologies, Ann Arbor, MI 48105 USA.

K. Sarabandi is with Radiation Laboratory, Department of Electrical Engineering and Computer Science, The University of Michigan, Ann Arbor, MI 48109 USA.

Publisher Item Identifier S 0018-926X(99)04831-0. and collision warning applications of millimeter-wave radars. Characterization of the scattering response of road surfaces under different conditions is pursued both theoretically and experimentally. To fully exploit the properties of the radar backscatter for a given application, techniques offered by radar polarimetry can be used to enhance the performance. To this end, extensive polarimetric backscatter measurements have been conducted at $W$-band for different types of road surfaces over a wide range of incidence angles. Asphalt, concrete, and gravel surfaces with varying degrees of surface roughness and wetness were observed.

Recently, a model for prediction of the backscatter response of smooth asphalt surfaces under various weather conditions (covered with water or ice) was developed [1]. This hybrid scattering model is appropriate for dense random media and is based on vector radiative transfer (RT) theory, where the fundamental quantities of the RT model are characterized by measurement. The application of the model is now extended to predict the backscatter response of smooth concrete surfaces (dry and ice- or water-covered).

Existence of a second layer of scatterers such as snow necessitates construction of a different volume scattering model for snow-covered road surfaces which is also discussed in this paper. The phase and extinction matrices of fresh snow layer can be computed using single scattering theory. Noting the azimuthal symmetry of snow particle distribution, ice particles in the snow medium are modeled as small spherical particles having the same volume as that of the snow flakes. The backscatter response of the snow-covered smooth asphalt or concrete surfaces is then computed from the superposition of the volume scattering from the snow layer and the underlying layer since the incoherent scattering from these media are statistically uncorrelated.

For asphalt or concrete roads with rough interface, the surface scattering component must be accounted for as well. Experimental results indicate that surface scattering becomes significant or even dominant when the surface roughness parameters are of the order of a wavelength. To develop a comprehensive scattering model for rough road surfaces, a surface scattering model known as the integral equation method (IEM) is incorporated into the volume scattering model previously developed for roads with smooth interface. IEM is a surface scattering model that accounts for multiple scattering up to second order and is expected to be valid for rough surfaces with low to moderate rms slope. Since the volumetric backscatter response is a gentle function of incidence angle, the surface roughness, which causes small variations in the 
transmitted ray direction, does not affect the volume scattering contribution significantly. Therefore, the volume scattering and the surface scattering responses can simply be added to predict the backscatter response of rough road surfaces.

This paper is organized as follows. First, the effective dielectric constants of concrete mixtures and snow are determined experimentally and theoretically, respectively. Next, the procedures for the characterization of ground-truth data such as surface roughness parameters are given. Section III briefly reviews the hybrid scattering model for dense random media with smooth surfaces. The procedure leading to the final formulation is outlined. The model for a multilayer random medium with two volume scattering layers is formulated for the snow-covered smooth surfaces. The surface scattering model is briefly described in Section III-C. The experimental procedure for the backscatter measurements and examination of the validity of the theoretical models are given in Section IV.

\section{Characterization of Ground-Truth Data}

Determination of influential parameters of the scattering medium is an essential step toward the development of scattering models. For example, characterization of the scattering behavior of road surfaces requires knowledge of the effective dielectric constants of pavement mixtures and possible surface cover such as water, ice, or snow. In this section, the effective dielectric constants of concrete mixtures and snow are examined. First, the effective dielectric constant of concrete mixtures is approximated by both high-frequency and low-frequency approaches. Then the simplified Polder-Van Santen mixing formula is used to calculate the effective dielectric constant of a snow medium. The measurement of the surface roughness statistics of rough interfaces between different media is also very important. The rms height and the correlation length are the most important surface parameters for modeling surface scattering. Both parameters are characterized experimentally.

\section{A. Effective Dielectric Constant of Concrete Mixtures}

A concrete mixture is a composite material commonly used for road pavement. The primary ingredients are cement, sand, and rocks. The theoretical approaches used for determining the effective dielectric constant of composite materials are generally complicated and require accurate statistical parameters of the medium such as permittivity fluctuation and correlation function [2], [3]. Here, an experimental approach is adopted since the constituents, their volume fraction and distribution are fixed for concrete mixtures used in road constructions. Both a low-frequency method and a high-frequency method are presented. An $L$-band ring resonator is used to perform a preliminary examination. Placement of the sample against the ring resonator elicits changes in the resonant frequency $(\Delta f)$ and the quality factor $(\Delta Q)$ from which the effective dielectric constant is inferred [4]. Ten concrete samples prepared by Midwest Testing Laboratory were measured, ten times each, and the average real part of the effective dielectric constant is found to be $4.54 \pm 0.15$.
The high-frequency approach for measuring the effective dielectric constant at $W$-band is accomplished by an inverse scattering algorithm presented in [1]. The radar cross section of thin cylindrical discs measured at normal incidence is used to calculate the effective dielectric constant at $94 \mathrm{GHz}$. Multiple reflections between the two ends of the cylindrical disc are found to be negligible by comparing the radar cross sections of the cylindrical concrete disc with and without a metal disc attached to its back. The procedure is repeated for discs of different thicknesses. The radar cross section of the concrete disc is compared with that of a circular metallic plate of the same area. The Fresnel reflection coefficient at normal incidence is calculated by finding the ratio of RCS of the concrete disc to that of the metallic disc. Due to the low-loss tangent of the concrete mixture, the real part of the effective dielectric constant can be evaluated from

$$
\epsilon_{c}^{\prime}=\left(\frac{1+\Gamma_{\circ}}{1-\Gamma_{\circ}}\right)^{2}
$$

where $\Gamma_{0}$ is the Fresnel reflection coefficient. The difference in the measured RCS's was shown to be $9.3 \pm 0.1 \mathrm{~dB}$. The average value of $\epsilon_{c}^{\prime}$ for ten concrete samples was calculated from (1) as $4.17 \pm 0.1$ which is close to the value estimated by the $L$-band ring resonator.

Measurement of the imaginary part of the effective dielectric constant $\left(\epsilon_{c}^{\prime \prime}\right)$ is often accomplished by observation of the RCS change in response to the attachment of the metallic disc to the back of the sample. $\epsilon_{c}^{\prime \prime}$ can be calculated in terms of the thickness of the disc and the change in the measured RCS values. Unfortunately, even the thinnest sample (2.54 $\mathrm{cm})$ exhibited no changes. Therefore, this method cannot provide a solution to calculate $\epsilon_{c}^{\prime \prime}$. However, the result suggests that concrete mixtures thicker than $5 \mathrm{~cm}$ can be considered semi-infinite. The $L$-band ring resonator provides an estimate $\epsilon_{c}^{\prime \prime}=0.36 \pm 0.05$.

\section{B. Effective Dielectric Constant of Snow}

The dielectric constant measurement of snow requires separate treatments for dry snow and wet snow. The case considered here is fresh dry snow. Modeling snow flakes with equivalent spherical ice particles results in particle sizes that fall within the Rayleigh region. Hence the dielectric constant $\left(\epsilon_{s}\right)$ of dry snow can be calculated by the simplified Polder-Van Santen mixing formula for spherical inclusions. With air as the host dielectric material and ice crystals as the inclusions, the real part and imaginary part of $\epsilon_{s}$ can be obtained from [5]

$$
\epsilon_{s}^{\prime}=(1+0.47 v)^{3} \quad \epsilon_{s}^{\prime \prime}=3 v \epsilon_{i}^{\prime \prime} \frac{\left(\epsilon_{s}^{\prime}\right)^{2}\left(2 \epsilon_{s}^{\prime}+1\right)}{\left(\epsilon_{i}^{\prime}+2 \epsilon_{s}^{\prime}\right)\left(\epsilon_{i}^{\prime}+2\left(\epsilon_{s}^{\prime}\right)^{2}\right)}
$$

where $v$ is the volume fraction of snow. The dielectric constant of ice is chosen to be $\epsilon_{i}=3.15+j 0.025$ according to the temperature of $-20{ }^{\circ} \mathrm{C}$ during the measurement [5]. For a volume fraction of 0.0742 (obtained from the ground-truth measurement of the snow-covered smooth asphalt surface), the effective dielectric constant of the snow is found to be $\epsilon_{s}=1.11+j 0.00074$. 
TABLE I

The Surface Roughness Statistics of the RoAd SURFACES EXAMINED IN THIS PAPER

\begin{tabular}{c|c|c}
\hline Road Surfaces & $s(\mathrm{~mm})$ & $\ell(\mathrm{mm})$ \\
\hline Smooth Asphalt & 0.34 & 4.2 \\
\hline Smooth Concrete & 0.2 & 38.6 \\
\hline Rough Asphalt & 0.9 & 6.3 \\
\hline
\end{tabular}

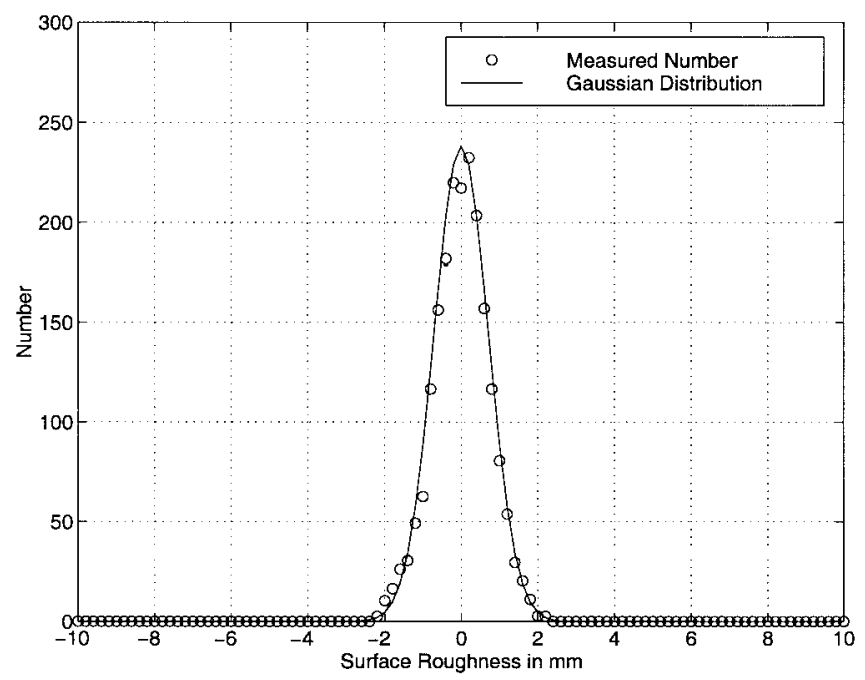

Fig. 1. The surface roughness distribution of the rough asphalt surface.

\section{Surface Roughness Statistics}

Surface roughness statistics are described by two important factors: the rms height of surface roughness $(s)$ and the surface autocorrelation function $(\rho(r))$. For one-dimensional surface profile measurements, $s$ is obtained from discrete height samples $h_{i}\left(x_{i}\right)$ at an appropriate spacing $\Delta x$, normally chosen such that $\Delta x \leq 0.1 \lambda$. The normalized autocorrelation function $\left(\left\langle h(x) h\left(x-x^{\prime}\right)\right\rangle\right)$ at a spatial displacement $x^{\prime}=$ $(j-1) \Delta x$ for the discrete case can be computed from

$$
\rho\left(x^{\prime}\right)=\frac{\sum_{i=1}^{N+1-j} h_{i} h_{i+j-1}}{\sum_{i=1}^{N} h_{i}^{2}}
$$

and the surface correlation length $(\ell)$ is defined as the displacement $x^{\prime}$ for which $\rho\left(x^{\prime}\right)$ is equal to $1 / e$ [6].

The surface roughness statistics of the rough asphalt surface considered in this study were measured by a laser ranging system with a range resolution of $0.1 \mathrm{~mm}$. Surface profiles from ten different locations were collected with each linear trace being at least $40 \mathrm{~cm}$ long. The horizontal resolution was set to be $0.2 \mathrm{~mm}$ which satisfies the requirement $\Delta r \leq 0.1 \lambda$. The measured $s$ and $\ell$ for the rough asphalt surface are listed in Table I.

For most road surfaces, the surface roughness exhibits a Gaussian height distribution with zero mean and standard deviation equal to $s$. This characteristic simplifies the analytical formulation of surface scattering model. Fig. 1 compares the measured surface roughness histogram of the rough asphalt surface with a Gaussian probability density function. The nor-

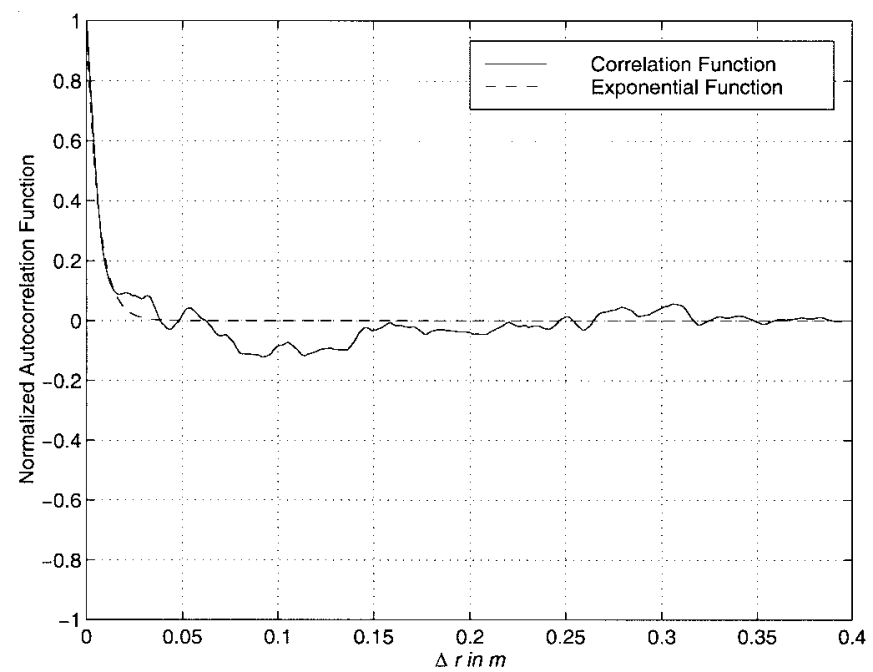

Fig. 2. The normalized autocorrelation function and the exponential approximation.

malized autocorrelation function of the rough asphalt surface is illustrated in Fig. 2 along with an approximate exponential function $\rho(r)=e^{-\frac{r}{\ell}}$.

\section{TheORETICAL ANALYSIS}

The backscatter response of road surfaces comes from two major sources: the volume scattering from pavement mixtures and the surface scattering from the rough interface between the air and the pavement mixture. The volume scattering is best described by the vector radiative transfer theory which is governed by the vector integro-differential equation

$$
\frac{\boldsymbol{I}(\hat{s}, \boldsymbol{r})}{d s}=-\kappa \boldsymbol{I}(\hat{s}, \boldsymbol{r})+\iint_{4 \pi} \boldsymbol{P}\left(\hat{s}, \hat{s}^{\prime}\right) \boldsymbol{I}\left(\hat{s}^{\prime}, \boldsymbol{r}\right) d \Omega^{\prime}
$$

where $I$ is an unknown $4 \times 1$ vector specific intensity, $\kappa$ is the $4 \times 4$ extinction matrix, and $\boldsymbol{P}$ is the $4 \times 4$ phase matrix. The bistatic scattering coefficient can be defined in terms of the incident specific intensity $\boldsymbol{I}^{i}$ and the scattered specific intensity $I^{s}$. For a $\beta$-polarized incident plane wave scattered as an $\alpha$ polarized spherical waves, the bistatic scattering coefficient $\sigma_{\alpha \beta}^{\circ}$ can be written as [3]

$$
\sigma_{\alpha \beta}^{\circ}\left(\pi-\theta_{0}, \phi_{0} ; \theta_{s}, \phi_{s}\right)=\frac{4 \pi \cos \theta_{s} I_{\alpha}^{s}\left(\theta_{s}, \phi_{s}\right)}{I_{\beta}^{i}\left(\pi-\theta_{0}, \phi_{0}\right)} .
$$

For the backscatter direction $\theta_{s}=\theta_{0}$ and $\phi_{s}=\pi+\phi_{0}$.

The standard first-order iterative solution of the RT equation requires separation of the specific intensity vector into upwardgoing $\left[\boldsymbol{I}^{+}(\theta, \phi, z)\right]$ and downward-going $\left[\boldsymbol{I}^{-}(\pi-\theta, \phi, z)\right]$ components. This method is incorporated with the appropriate boundary conditions to establish the relation between the upward- and downward-going intensities. The iterative solution has been described by many authors [3], [5], [7], and will not be discussed here. Quantities such as the extinction and phase matrices are related to the physical parameters of the medium such as particle size, density, permittivity, and 


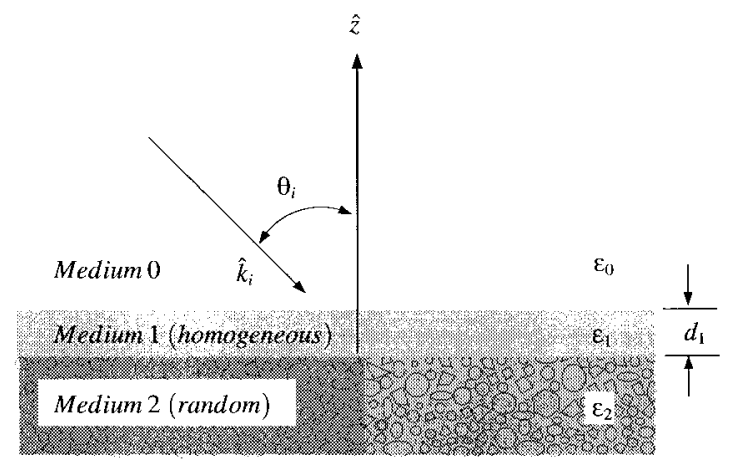

Fig. 3. The geometry of the scattering from a multiple-layer medium with one volume scattering layer.

orientation distribution. For dense random media, such as pavement mixtures, these quantities are difficult to characterize analytically and are obtained experimentally. On the other hand, the extinction and phase matrices of snow can be described precisely by the Rayleigh scattering model.

To analyze the backscatter response of rough road surfaces, the effect of surface scattering cannot be ignored. Many approximate analytical solutions of the surface scattering problems have been introduced. The choice of the appropriate model depends on the normalized rms height $(k s)$ and correlation length $(k \ell)$. For surfaces with small rms roughness and slope, the small perturbation model (SPM) is often used [8], [9]. The Kirchhoff approximation (KA) is usually used for surfaces with relatively small slopes and large radii of curvature [10]. Other techniques, such as the phase perturbation method (PPM) [11] and the unified perturbation expansion (UPE) [12], are mainly used in the low-frequency regime. To investigate the backscatter behavior of a rough asphalt surface at $W$-band, the IEM technique is selected. In the following, the scattering solutions for three different road surfaces are discussed.

\section{A. Backscatter from Concrete Surfaces}

The hybrid scattering model used for the backscatter response of smooth asphalt surfaces can also be applied for concrete surfaces. Basically, this model is appropriate for a half-space random medium covered possibly with a multilayer homogeneous medium as shown in Fig. 3. The homogeneous layer can be ice, water, or combination depending on the weather conditions considered. The procedures leading to the final formulation of this model will be reviewed here for convenience.

The experiment conducted for measuring $\epsilon_{c}^{\prime \prime}$ indicates that the concrete layer can be considered semi-infinite which simplifies the boundary conditions of the existing problem. The boundary conditions related to the present problem are

$$
\begin{aligned}
\boldsymbol{I}^{-}\left(\pi-\theta_{2}, \phi_{0}, 0^{-}\right)= & \boldsymbol{T}_{02} \boldsymbol{I}^{i}\left(\pi-\theta_{0}, \phi_{0}, d_{1}^{+}\right) \\
& \times \delta\left(\theta-\theta_{0}\right) \delta\left(\phi-\phi_{0}\right) \\
\boldsymbol{I}^{s}\left(\theta_{0}, \phi_{0}, d_{1}^{+}\right)= & \boldsymbol{T}_{20} \boldsymbol{I}^{+}\left(\theta_{2}, \phi_{0}, 0^{-}\right) .
\end{aligned}
$$

$\boldsymbol{T}_{02}$ is the transmissivity matrix given in [1, eq. (9)].
The assumption of an isotropic random medium for concrete mixtures reduces the extinction matrix $\kappa_{c}$ to a scalar quantity $\left(\kappa_{c}\right)$ and simplifies the phase matrix $\boldsymbol{P}_{c}$ to the following form:

$$
\boldsymbol{P}_{c}=\left[\begin{array}{cccc}
p_{1, c} & p_{2, c} & 0 & 0 \\
p_{2, c} & p_{1, c} & 0 & 0 \\
0 & 0 & p_{3, c}+p_{2, c} & -p_{4, c} \\
0 & 0 & p_{4, c} & p_{3, c}-p_{2, c}
\end{array}\right]
$$

where the subscript $c$ denotes concrete.

The first-order iterative solution of the RT equation subject to the aforementioned boundary equations and the assumption of an isotropic random medium results in the following expression for the backscattered specific intensity:

$$
\boldsymbol{I}^{s}\left(\theta_{0}, \phi_{0}+\pi, d_{1}^{+}\right)=\frac{1}{2 \kappa_{e, c}} \boldsymbol{T}_{20} \boldsymbol{P}_{c} \boldsymbol{T}_{02} \boldsymbol{I}^{i}\left(\pi-\theta_{0}, \phi_{0}, d_{1}^{+}\right) .
$$

Combining (5) and (8), the backscattering coefficients are

$$
\begin{aligned}
& \sigma_{v v}^{\circ}=4 \pi \cos \theta_{0}\left|t_{02}^{v}\right|^{2}\left|t_{20}^{v}\right|^{2} \frac{p_{1, c}}{2 \kappa_{e, c}} \\
& \sigma_{h h}^{\circ}=4 \pi \cos \theta_{0}\left|t_{02}^{h}\right|^{2}\left|t_{20}^{h}\right|^{2} \frac{p_{1, c}}{2 \kappa_{e, c}} \\
& \sigma_{v h}^{\circ}=4 \pi \cos \theta_{0}\left|t_{02}^{h}\right|^{2}\left|t_{20}^{v}\right|^{2} \frac{p_{2, c}}{2 \kappa_{e, c}} \\
& \sigma_{h v}^{\circ}=4 \pi \cos \theta_{0}\left|t_{02}^{v}\right|^{2}\left|t_{20}^{h}\right|^{2} \frac{p_{2, c}}{2 \kappa_{e, c}}
\end{aligned}
$$

where $t_{02}^{v, h}$ is the Fresnel transmission coefficient from medium 0 to medium 2 , given by [1, eq. (10)]. Instead of determining the values of $\frac{p_{1, c}}{2 \kappa_{e, c}}$ and $\frac{p_{2, c}}{2 \kappa_{e, c}}$ in terms of particle size, shape, density, and permittivity, the quantitative characterization was accomplished by comparing the measured backscatter data at one angle under dry condition to (9). The results are used for other incidence angles and physical conditions.

The statistics of phase difference are also of interest in active remote sensing. Noting that the phase difference statistics are sensitive to physical parameters of the target, the phase difference parameters are also modeled theoretically. The statistics of phase difference can be obtained from the Mueller matrix [13]. The probability density function of the phase difference $\left(\phi_{h h}-\phi_{v v}\right)$ can be characterized in terms of two parameters: the degree of correlation $(\alpha)$ and the mean phase difference $(\zeta)$. The degree of correlation and the mean phase difference can be expressed in terms of the elements of the phase matrix $p_{i, c}[1]$. The expressions are given by

$$
\alpha=\sqrt{\frac{p_{3, c}^{2}+p_{4, c}^{2}}{p_{1, c}^{2}}}
$$

and

$$
\zeta=\tan ^{-1} \frac{p_{4, c}}{p_{3, c}}+\angle\left(t_{20}^{v} t_{20}^{h *}\right)+\angle\left(t_{02}^{v} t_{02}^{h *}\right) .
$$

\section{B. Backscatter from Snow-Covered Smooth Surfaces}

Scattering from snow-covered road surfaces with a smooth interface can be treated as a scattering problem of a two-layer 


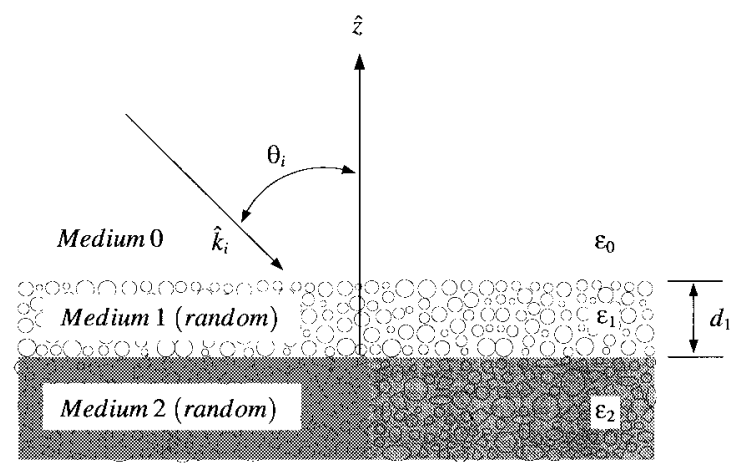

Fig. 4. The geometry of the scattering from a multiple-layer medium with two volume scattering layers.

random medium. The geometry of this problem is shown in Fig. 4. Consider the case of snow-covered smooth asphalt surfaces. As mentioned before, the underlying asphalt layer can be considered semi-infinite, which reduces the boundary conditions to the following relatively simple forms:

$$
\begin{aligned}
& \boldsymbol{I}^{-}\left(\pi-\theta_{1}, \phi_{0}, d_{1}^{-}\right) \\
& \quad=T_{01} \boldsymbol{I}^{i}\left(\pi-\theta_{0}, \phi_{0}, d_{1}^{+}\right) \delta\left(\theta-\theta_{0}\right) \delta\left(\phi-\phi_{0}\right) \\
& \quad+\boldsymbol{R}_{10} \boldsymbol{I}^{+}\left(\theta_{1}, \phi_{0}, d_{1}^{-}\right) \\
& \quad \boldsymbol{I}^{+}\left(\theta_{1}, \phi_{0}, 0^{+}\right) \\
& \quad=\boldsymbol{R}_{12} \boldsymbol{I}^{-}\left(\pi-\theta_{1}, \phi_{0}, 0^{+}\right)+\boldsymbol{T}_{21} \boldsymbol{I}^{+}\left(\theta_{2}, \phi_{0}, 0^{-}\right) \\
& \boldsymbol{I}^{-}\left(\pi-\theta_{2}, \phi_{0}, 0^{-}\right) \\
& \quad=\boldsymbol{T}_{12} \boldsymbol{I}^{-}\left(\pi-\theta_{1}, \phi_{0}, 0^{+}\right)+\boldsymbol{R}_{21} \boldsymbol{I}^{+}\left(\theta_{2}, \phi_{0}, 0^{-}\right) .
\end{aligned}
$$

Here $\boldsymbol{R}_{10}$ is the reflectivity matrix given by

$$
\boldsymbol{R}_{10}=\left[\begin{array}{cccc}
\left|r_{10}^{v}\right|^{2} & 0 & 0 & 0 \\
0 & \left|r_{10}^{h}\right|^{2} & 0 & 0 \\
0 & 0 & \operatorname{Re}\left(r_{10}^{v} r_{10}^{h *}\right) & -\operatorname{Im}\left(r_{10}^{v} r_{10}^{h *}\right) \\
0 & 0 & \operatorname{Im}\left(r_{10}^{v} r_{10}^{h *}\right) & \operatorname{Re}\left(r_{10}^{v} r_{10}^{h *}\right)
\end{array}\right]
$$

where $r_{10}^{v}$ and $r_{10}^{h}$, the $v$-polarized and $h$-polarized Fresnel reflection coefficients at the interface of medium 1 and medium 0 . The matrices $\boldsymbol{R}_{12}$ and $\boldsymbol{R}_{21}$ have a form similar to $\boldsymbol{R}_{10}$ using appropriate subscripts. $\boldsymbol{T}_{01}, \boldsymbol{T}_{12}$, and $\boldsymbol{T}_{21}$ have the same form as $\boldsymbol{T}_{02}$ except for the change in the subscript. $t_{01}^{v}$ and $t_{01}^{h}$ are the $v$-polarized and $h$-polarized field transmission coefficients at the interface of medium 0 and medium 1 . The field transmission coefficients at the interface of adjacent medium $m$ and $n$ are given by

$$
t_{m n}^{v}=\left(1+r_{m n}^{v}\right) \frac{\eta_{n}}{\eta_{m}} \quad t_{m n}^{h}=1+r_{m n}^{h} .
$$

The scattering behavior of snow, unlike the pavement material, has been modeled successfully and reported in many publications [3], [5]. The collected ground truth shows that the average radius $a$ of the ice crystals for the experiment conducted for the snow-covered asphalt surface was around $0.25 \mathrm{~mm}$. Therefore, Rayleigh scattering model can be used $(k a=0.5)$ to predict the phase and extinction matrices of the snow layer. The phase matrix $\boldsymbol{P}_{s}$ of the Rayleigh scatterer has the following form:

$$
\boldsymbol{P}_{s}\left(\theta^{s}, \phi^{s} ; \theta^{i}, \phi^{i}\right)=\left[\begin{array}{cccc}
p_{11} & p_{12} & p_{13} & 0 \\
p_{21} & p_{22} & p_{23} & 0 \\
p_{31} & p_{32} & p_{33} & 0 \\
0 & 0 & 0 & p_{44}
\end{array}\right]
$$

where the subscript $s$ denotes snow. The expressions for each element of $\boldsymbol{P}_{s}$ are presented in [3] and [5] and are not repeated here. The extinction coefficient for snow $\kappa_{e, s}$ is the sum of the scattering coefficient $\kappa_{s, s}$ and the absorption coefficient $\kappa_{a, s}$. The scattering coefficient $\kappa_{s, s}$ is given by

$$
\kappa_{s, s}=2 v k^{4} a^{3}\left|\frac{\epsilon_{s}-\epsilon}{\epsilon_{s}+2 \epsilon}\right|^{2}
$$

where $v$ is the volume fraction of the scatterers, $\epsilon_{s}$ is the dielectric constant of the ice particles, $\epsilon$ is the dielectric constant of the background material, and $k$ is the wavenumber in the background material. The absorption coefficient $\kappa_{a, s}$ is given by

$$
\kappa_{a, s}=v k \frac{\epsilon_{s}^{\prime \prime}}{\epsilon}\left|\frac{3 \epsilon}{\epsilon_{s}+2 \epsilon}\right|^{2}
$$

The copolarized backscatter response of the snow-covered smooth asphalt surface can be solved by the first-order iterative solution of the RT equation subject to the boundary conditions in (12). The backscattered specific intensity can be related to the incident specific intensity by

$$
\begin{aligned}
\boldsymbol{I}^{s}\left(\theta_{0}, \phi_{0}+\pi, d_{1}^{+}\right) \\
=\frac{1}{\cos \theta_{1}} \boldsymbol{T}_{10} \boldsymbol{F}^{+}\left[\boldsymbol{P}_{s}\left(\theta_{1}, \phi_{1}+\pi ; \theta_{1}, \phi_{1}\right) \boldsymbol{F}^{+} \boldsymbol{R}_{12} \boldsymbol{T}_{01} \boldsymbol{I}_{0}^{i}\right. \\
\quad \times e^{\frac{-2 \kappa e, s d_{1}}{\cos \theta_{1}}} d_{1}+\boldsymbol{P}_{s}\left(\theta_{1}, \phi_{1}+\pi ; \pi-\theta_{1}, \phi_{1}\right) \boldsymbol{F}^{-} \boldsymbol{T}_{01} \boldsymbol{I}_{0}^{i} \\
\left.\quad \times \frac{\cos \theta_{1}}{2 \kappa_{e, s}}\left(1-e^{\frac{-2 \kappa e, s d_{1}}{\cos \theta_{1}}}\right)\right]+\frac{1}{\cos \theta_{1}} \boldsymbol{T}_{10} \boldsymbol{F}^{+} \boldsymbol{R}_{12} e^{\frac{-2 \kappa, s d_{1}}{\cos \theta_{1}}} \\
\quad \times\left[\boldsymbol{P}_{s}\left(\pi-\theta_{1}, \phi_{1}+\pi ; \theta_{1}, \phi_{1}\right) \boldsymbol{F}^{+} \boldsymbol{R}_{12} \boldsymbol{T}_{01} \boldsymbol{I}_{0} \frac{\cos \theta_{1}}{2 \kappa_{e, s}}\right. \\
\quad \times\left(1-e^{\frac{-2 \kappa e, s d_{1}}{\cos \theta_{1}}}\right)+\boldsymbol{P}_{s}\left(\pi-\theta_{1}, \phi_{1}+\pi ; \pi-\theta_{1}, \phi_{1}\right) \\
\left.\quad \times \boldsymbol{F}^{-} \boldsymbol{T}_{01} \boldsymbol{I}_{0}^{i} d_{1}\right]+e^{\frac{-2 \kappa_{e}, s d_{1}}{\cos \theta_{1}}} \boldsymbol{T}_{10} \boldsymbol{F}^{+} \boldsymbol{T}_{21} \boldsymbol{P}_{a} \\
\quad \times\left(\theta_{2}, \phi_{2}+\pi ; \pi-\theta_{2}, \phi_{2}\right) \boldsymbol{T}_{12} \boldsymbol{F}^{-} \boldsymbol{T}_{01} \boldsymbol{I}_{0}^{i} \frac{1}{2 \kappa_{e, a}}
\end{aligned}
$$

where the subscript $a$ denotes the quantities of asphalt mixture. The expressions for $\boldsymbol{F}^{+}$and $\boldsymbol{F}^{-}$are given by

$$
\begin{aligned}
& \boldsymbol{F}^{+}=\left(\overline{\boldsymbol{I}}-\boldsymbol{R}_{12} \boldsymbol{R}_{10} e^{\frac{-2 \kappa_{e, s} d_{1}}{\cos \theta_{1}}}\right)^{-1} \\
& \boldsymbol{F}^{-}=\left(\overline{\boldsymbol{I}}-\boldsymbol{R}_{10} \boldsymbol{R}_{12} e^{\frac{-2 \kappa_{e, s} d_{1}}{\cos \theta_{1}}}\right)^{-1}
\end{aligned}
$$

and $\bar{I}$ is a $4 \times 4$ unitary matrix. Equation (18) indicates that the backscatter response of the snow-covered smooth asphalt surface consists of the volume scattering from the snow layer and the volume scattering from the underlying asphalt layer. The backscattering coefficients for the snow layer, based on 
the first-order iterative solution of the RT equation, can be derived from (5) and (18) and expressed as

$$
\begin{aligned}
\sigma_{v v, s}^{\circ(1)}= & \frac{3 \kappa_{s, s}}{2} \cos \theta_{0}\left|t_{10}^{v}\right|^{2}\left|t_{01}^{v}\right|^{2} F_{11}^{2} \\
\times & {\left[\frac{2 d_{1}}{\cos \theta_{1}}\left(\sin ^{2} \theta_{1}-\cos ^{2} \theta_{1}\right)^{2}\left|r_{12}^{v}\right|^{2} e^{\frac{-2 \kappa_{e}, s d_{1}}{\cos \theta_{1}}}\right.} \\
& \left.+\frac{1}{2 \kappa_{e, s}}\left(1-e^{\frac{-2 \kappa_{e}, s d_{1}}{\cos \theta_{1}}}\right)\left(1+\left(\left|r_{12}^{v}\right|^{2}\right)^{2} e^{\frac{-2 \kappa_{e} d_{1}}{\cos \theta_{1}}}\right)\right] \\
\sigma_{h h, s}^{\circ(1)}= & \frac{3 \kappa_{s, s}}{2} \cos \theta_{0}\left|t_{10}^{h}\right|^{2}\left|t_{01}^{h}\right|^{2} F_{22}^{2}\left[\frac{2 d_{1}}{\cos \theta_{1}}\left|r_{12}^{h}\right|^{2} e^{\frac{-2 \kappa_{e}, s}{\cos \theta_{1}}}\right. \\
& \left.+\frac{1}{2 \kappa_{e, s}}\left(1-e^{\frac{-2 \kappa_{e}, s d_{1}}{\cos \theta_{1}}}\right)\left(1+\left(\left|r_{12}^{h}\right|^{2}\right)^{2} e^{\frac{-2 \kappa_{e, s} d_{1}}{\cos \theta_{1}}}\right)\right] \\
\sigma_{v h, s}^{\circ(1)}= & 0 \\
\sigma_{h v, s}^{\circ(1)}= & 0
\end{aligned}
$$

where the superscript (1) denotes the first-order iterative solution. $F_{11}$ and $F_{22}$ are given by

$$
\begin{aligned}
& F_{11}=\left(1-\left|r_{10}^{v}\right|^{2}\left|r_{12}^{v}\right|^{2} e^{\frac{-2 \kappa_{e}, s d_{1}}{\cos \theta_{1}}}\right)^{-1} \\
& F_{22}=\left(1-\left|r_{10}^{h}\right|^{2}\left|r_{12}^{h}\right|^{2} e^{\frac{-2 \kappa_{e}, s d_{1}}{\cos \theta_{1}}}\right)^{-1} .
\end{aligned}
$$

As given by (20), the first-order cross-polarized backscatter response predicted by the RT model is zero. Hence higher order solutions must be considered for determining the crosspolarized response. The cross-polarized backscattering coefficient derived from the second-order iterative solution is given by [3]

$$
\begin{aligned}
\sigma_{h v, s}^{\circ(2)}= & 4 \frac{\cos \theta_{0}}{\cos \theta_{1}}\left(\frac{3 \kappa_{s, s}}{8}\right)^{2}\left|t_{10}^{h}\right|^{2}\left|t_{01}^{v}\right|^{2} F_{22} F_{11} \int_{0}^{\pi / 2} d \theta^{\prime} \frac{\sin \theta^{\prime}}{\cos \theta^{\prime}} \\
& \times\left[Q_{1}\left(\theta_{1}, \theta^{\prime}\right)+Q_{2}\left(\theta_{1}, \theta^{\prime}\right)+Q_{3}\left(\theta_{1}, \theta^{\prime}\right)\right]
\end{aligned}
$$

where the superscript (2) denotes the second-order iterative solution. The expressions for $Q_{1}\left(\theta_{1}, \theta^{\prime}\right), Q_{2}\left(\theta_{1}, \theta^{\prime}\right)$, and $Q_{3}\left(\theta_{1}, \theta^{\prime}\right)$ are given in the paper by Shin and Kong [14]. $\sigma_{v h, s}^{\circ(2)}$ can be obtained from $\sigma_{h v, s}^{\circ(2)}$ using the reciprocity theorem.

The expressions for the backscattering coefficients of the underlying asphalt layer can also be deduced from (5) and (18). Since the phase matrix of the asphalt mixture $\boldsymbol{P}_{a}$ has the same form as $\boldsymbol{P}_{c}$, the backscattering coefficients can be expressed as

$$
\begin{aligned}
\sigma_{v v, a}^{\circ(1)}= & 4 \pi \cos \theta_{0}\left|t_{10}^{v}\right|^{2}\left|t_{21}^{v}\right|^{2}\left|t_{12}^{v}\right|^{2}\left|t_{01}^{v}\right|^{2} F_{11}^{2} e^{\frac{-2 \kappa_{e}, d_{1}}{\cos \theta_{1}}} \frac{p_{1, a}}{2 \kappa_{e, a}} \\
\sigma_{h h, a}^{\circ(1)}= & 4 \pi \cos \theta_{0}\left|t_{10}^{h}\right|^{2}\left|t_{21}^{h}\right|^{2}\left|t_{12}^{h}\right|^{2}\left|t_{01}^{h}\right|^{2} F_{22}^{2} e^{\frac{-2 \kappa_{e}, s d_{1}}{\cos \theta_{1}}} \frac{p_{1, a}}{2 \kappa_{e, a}} \\
\sigma_{v h, a}^{\circ(1)}= & 4 \pi \cos \theta_{0}\left|t_{10}^{v}\right|^{2}\left|t_{21}^{v}\right|^{2}\left|t_{12}^{h}\right|^{2}\left|t_{01}^{h}\right|^{2} \\
& \times F_{11} F_{22} e^{\frac{-2 \kappa_{e, s} d_{1}}{\cos \theta_{1}}} \frac{p_{2, a}}{2 \kappa_{e, a}} \\
\sigma_{h v, a}^{\circ(1)}= & 4 \pi \cos \theta_{0}\left|t_{10}^{h}\right|^{2}\left|t_{21}^{h}\right|^{2}\left|t_{12}^{v}\right|^{2}\left|t_{01}^{v}\right|^{2} \\
& \times F_{22} F_{11} e^{\frac{-2 \kappa_{e, s} d_{1}}{\cos \theta_{1}}} \frac{p_{2, a}}{2 \kappa_{e, a}} .
\end{aligned}
$$

The quantities $\frac{p_{1, a}}{2 \kappa_{e, a}}$ and $\frac{p_{2, a}}{2 \kappa_{e, a}}$ are determined by the experimental data as in the case of $\frac{p_{1, c}}{2 \kappa_{e, c}}$ and $\frac{p_{2, c}}{2 \kappa_{e, c}}$.

The overall backscatter response of the snow-covered smooth asphalt surface is the sum of the response from the snow layer and the response from the asphalt layer

$$
\begin{aligned}
& \sigma_{v v}^{\circ}=\sigma_{v v, s}^{\circ(1)}+\sigma_{v v, a}^{\circ(1)} \\
& \sigma_{h h}^{\circ}=\sigma_{h h, s}^{\circ(1)}+\sigma_{h h, a}^{\circ(1)} \\
& \sigma_{v h}^{\circ}=\sigma_{v h, s}^{\circ(2)}+\sigma_{v h, a}^{\circ(1)} \\
& \sigma_{h v}^{\circ}=\sigma_{h v, s}^{\circ(2)}+\sigma_{h v, a}^{\circ(1)} .
\end{aligned}
$$

\section{Backscatter from Rough Asphalt Surfaces}

The measured backscattering coefficients of a rough asphalt surface with rms height $0.9 \mathrm{~mm}(k s=1.8)$ were compared with the results predicted by the volume scattering model developed for smooth asphalt surfaces [1]. The comparison indicated that the volume scattering cannot accurately predict the overall backscatter response of such a surface. That is, a comprehensive scattering model should also account for the surface scattering component. The backscatter behavior of a rough surface can be described in terms of its surface roughness statistics, namely, the rms height of the surface profile $s$ and the surface autocorrelation function $\rho(r)$, and the permittivity contrast between two media.

The effective dielectric constant of asphalt mixtures at $W$-band was found to be $3.18+j 0.1$ which presents a significant contrast to that of the upper medium (air). Hence, a considerable rough surface scattering contribution is expected. Noting that the permittivity fluctuation within the asphalt medium are relatively small and somewhat independent of surface roughness statistics, the surface scattering contribution can be computed independently of the volume scattering. In other words, to compute the surface scattering component the asphalt medium is considered a homogeneous medium with a dielectric constant of $\epsilon_{a}=3.18+j 0.1$.

Of the existing analytical models for rough surface scattering, the integral equation method (IEM) is considered as an advanced analytical solution. This method is adopted here for the calculation of the surface scattering contribution from rough asphalt surfaces. The formulation is obtained from a simplified second-order iterative physical optics (PO) approximation. Besides the term estimated by the standard Kirchhoff approximation, this formulation contains a complementary term to represent the second-order multiple scattering [15], that is,

$$
\begin{aligned}
\hat{n} \times \boldsymbol{E} & =(\hat{n} \times \boldsymbol{E})_{k}+(\hat{n} \times \boldsymbol{E})_{c} \\
\hat{n} \times \boldsymbol{H} & =(\hat{n} \times \boldsymbol{H})_{k}+(\hat{n} \times \boldsymbol{H})_{c}
\end{aligned}
$$

where the subscripts $k$ and $c$, respectively, denote the Kirchhoff and complementary components. Depending on the polarization, the Kirchhoff tangential fields can be approximated 
by the following expressions:

$$
\begin{aligned}
(\hat{n} \times \boldsymbol{E})_{k, v} & =\left(1-r^{v}\right) \hat{n} \times \hat{v} E^{i} \\
\eta(\hat{n} \times \boldsymbol{H})_{k, v} & =\left(1+r^{v}\right) \hat{n} \times\left(\hat{k}_{i} \times \hat{v}\right) E^{i} \\
(\hat{n} \times \boldsymbol{E})_{k, h} & =\left(1+r^{h}\right) \hat{n} \times \hat{h} E^{i} \\
\eta(\hat{n} \times \boldsymbol{H})_{k, h} & =\left(1-r^{h}\right) \hat{n} \times\left(\hat{k}_{i} \times \hat{h}\right) E^{i}
\end{aligned}
$$

where $r^{v}$ and $r^{h}$ are the Fresnel reflection coefficients, $E^{i}$ is the amplitude of the incident electric field, and $\hat{k}_{i}$ is the unit vector in the incident direction. Similarly, the approximate expressions for the complementary tangential fields are given by

$$
\begin{aligned}
(\hat{n} \times \boldsymbol{E})_{c, v} & \approx-\frac{1}{4 \pi} \hat{n} \times \int\left[\left(1-r^{v}\right) \overrightarrow{\mathcal{E}}_{v}+\left(1+r^{v}\right) \overrightarrow{\mathcal{E}}_{v t}\right] d s^{\prime} \\
(\hat{n} \times \boldsymbol{H})_{c, v} & \approx \frac{1}{4 \pi} \hat{n} \times \int\left[\left(1+r^{v}\right) \overrightarrow{\mathcal{H}}_{v}+\left(1-r^{v}\right) \overrightarrow{\mathcal{H}}_{v t}\right] d s^{\prime} \\
(\hat{n} \times \boldsymbol{E})_{c, h} & \approx-\frac{1}{4 \pi} \hat{n} \times \int\left[\left(1+r^{h}\right) \overrightarrow{\mathcal{E}}_{h}+\left(1-r^{h}\right) \overrightarrow{\mathcal{E}}_{h t}\right] d s^{\prime} \\
(\hat{n} \times \boldsymbol{H})_{c, h} & \approx \frac{1}{4 \pi} \hat{n} \times \int\left[\left(1-r^{h}\right) \overrightarrow{\mathcal{H}}_{h}+\left(1+r^{h}\right) \overrightarrow{\mathcal{H}}_{h t}\right] d s^{\prime}
\end{aligned}
$$

The expressions for $\overrightarrow{\mathcal{E}}_{p}, \overrightarrow{\mathcal{E}}_{p t}, \overrightarrow{\mathcal{H}}_{p}$, and $\overrightarrow{\mathcal{H}}_{p t}(p=v, h)$ are given by Fung [15].

In accordance with the Stratton-Chu integral, the scattered far field in the medium above the rough surface can be written as

$$
\boldsymbol{E}^{s}=\frac{j k}{4 \pi R} e^{j k R} \int\left[\hat{k}_{s} \times(\hat{n} \times \boldsymbol{E})+\eta(\hat{n} \times \boldsymbol{H})\right] e^{-j\left(\boldsymbol{k}_{s} \cdot \boldsymbol{r}\right)} d s
$$

where $k_{s}=k_{s x} \hat{x}+k_{s y} \hat{y}+k_{s z} \hat{z}$. Note that the $e^{-j \omega t}$ convention is adopted in (28). To calculate the backscattering coefficients, the incoherent scattered power must be determined. This is obtained by subtracting the coherent power from the total scattered power, that is,

$$
P_{p q}=\left\langle E_{p q}^{s} E_{p q}^{s *}\right\rangle-\left\langle E_{p q}^{s}\right\rangle\left\langle E_{p q}^{s}\right\rangle^{*} .
$$

Assuming a Gaussian surface roughness statistic, the averaging operation can be carried out analytically. The backscattering coefficient $\sigma_{p q}^{\circ}$ is related to the average power $P_{p q}$ by

$$
\sigma_{p q}^{\circ}=\left(4 \pi R^{2} P_{p q}\right) /\left(\left|E^{i}\right|^{2} A_{\text {illu }}\right)
$$

where $A_{\text {illu }}$ is the illumination area. The copolarized backscattering coefficients can be estimated from [15] (in backscatter $\left.k_{s z}=k_{z}, k_{s x}=-k_{x}\right)$

$$
\sigma_{p p}^{\circ}=\frac{k^{2}}{2} e^{-2 s^{2} k_{z}^{2}} \sum_{n=1}^{\infty} s^{2 n}\left|I_{p p}^{n}\right|^{2} \frac{W^{(n)}\left(-2 k_{x}, 0\right)}{n !}
$$

where $k_{x}$ and $k_{z}$ are the components of the incident propagation vector $\boldsymbol{k}_{i}=k_{x} \hat{x}+k_{y} \hat{y}-k_{z} \hat{z}$ and $W^{(n)}$ is the roughness spectrum of the surface and is related to the $n$th power of the surface autocorrelation function $\rho^{n}(r)$ by the Fourier transform as follows:

$$
\begin{aligned}
& W^{(n)}\left(k_{s x}-k_{x}, k_{s y}-k_{y}\right) \\
& \quad=\frac{1}{2 \pi} \int \rho^{n}(x, y) e^{j\left(k_{s x}-k_{x}\right) x+j\left(k_{s y}-k_{y}\right) y} d x d y
\end{aligned}
$$

and

$$
I_{p p}^{n}=\left(2 k_{z}\right)^{n} f_{p p} e^{-s^{2} k_{z}^{2}}+\frac{k_{z}^{n}\left[F_{p p}\left(-k_{x}, 0\right)+F_{p p}\left(k_{x}, 0\right)\right]}{2}
$$

where $f_{p p}$ and $F_{p p}(\cdot, \cdot)$ are the Kirchhoff and complementary field coefficients and are approximated by the following forms:

$$
\begin{aligned}
f_{v v}= & \frac{2 r^{v}}{\cos \theta_{0}} \\
f_{h h}= & -\frac{2 r^{h}}{\cos \theta_{0}} \\
F_{v v}\left(-k_{x}, 0\right)+F_{v v}\left(k_{x}, 0\right)= & \frac{2 \sin ^{2} \theta_{0}\left(1+r^{v}\right)^{2}}{\cos \theta_{0}}\left[\left(1-\frac{1}{\epsilon_{r}}\right)\right. \\
& \left.+\frac{\mu_{r} \epsilon_{r}-\sin ^{2} \theta_{0}-\epsilon_{r} \cos ^{2} \theta_{0}}{\epsilon_{r}^{2} \cos ^{2} \theta_{0}}\right] \\
F_{h h}\left(-k_{x}, 0\right)+F_{h h}\left(k_{x}, 0\right)= & -\frac{2 \sin ^{2} \theta_{0}\left(1+r^{h}\right)^{2}}{\cos \theta_{0}}\left[\left(1-\frac{1}{\mu_{r}}\right)\right. \\
& \left.+\frac{\mu_{r} \epsilon_{r}-\sin ^{2} \theta_{0}-\mu_{r} \cos ^{2} \theta_{0}}{\mu_{r}^{2} \cos ^{2} \theta_{0}}\right] .
\end{aligned}
$$

In the equations above, the local incidence angle in the Fresnel reflection coefficients, $r^{v}$ and $r^{h}$, is approximated by the global incidence angle.

\section{EXPERIMENTAL RESULTS}

In this section, the validity of the models developed in the previous sections is verified by experimental data. The results of the polarimetric backscatter measurements at $94 \mathrm{GHz}$ on the aforementioned surfaces are presented and compared with the theoretical values predicted by the models. The University of Michigan 94-GHz fully polarimetric radar was used to perform the backscatter measurements at near-grazing incidence angles (from $70^{\circ}$ to $88^{\circ}$ ). The radar is a network-analyzer-based system operated in a step frequency mode with a bandwidth of $2 \mathrm{GHz}$. A gimbal assembly was constructed and used to scan the distributed targets during the measurements. The frequency decorrelation behavior of the backscatter response of the distributed targets is expected to increase the number of independent samples [16]. At least 80 independent samples were used in the backscatter measurements. An external calibration technique was used to remove the systematic errors such as channel imbalances, antenna crosstalk, and the imperfection of polarization switches [17].

First the polarimetric backscatter response of a concrete surface is considered. The concrete surface used for this experiment has an rms height of $0.2 \mathrm{~mm}$ and a correlation length of $38.5 \mathrm{~mm}$. Since the rms height of the surface is much smaller than the wavelength, the rough surface contribution can be ignored. Two physical conditions are considered: 1) a dry concrete surface, and 2) a concrete surface covered with an ice layer $1.7 \mathrm{~mm}$ thick. The measured backscatter data at an incidence angle of $74^{\circ}$ under dry conditions were used to characterize the extinction and phase matrices of the concrete mixtures and are reported in Table II. It is found that volume scattering of concrete medium is less than that of asphalt (see Table II and Table IV). This result is not surprising as the 
TABLE II

Normalized Elements of Phase Matrix of Concrete Mixtures at $94 \mathrm{GHz}$ Obtained From the Polarimetric Backscatter Measurements

\begin{tabular}{c|c|c|c}
\hline$p_{1, c} / \kappa_{e, c}$ & $p_{2, c} / \kappa_{e, c}$ & $p_{3, c} / \kappa_{e, c}$ & $p_{4, c} / \kappa_{e, c}$ \\
\hline $6.4 \times 10^{-3}$ & $6.8 \times 10^{-4}$ & $3.4 \times 10^{-3}$ & $2.2 \times 10^{-3}$ \\
\hline
\end{tabular}

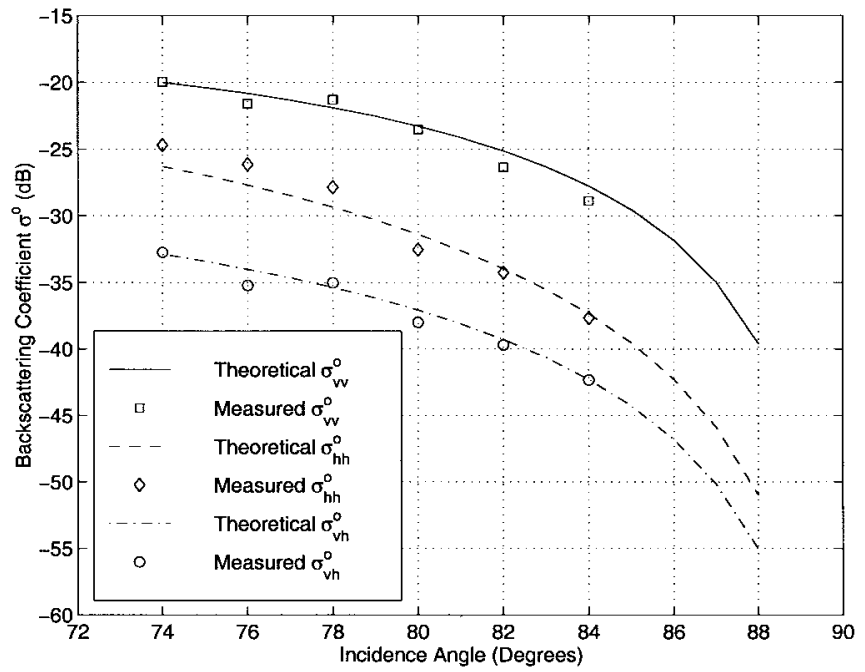

Fig. 5. The comparison between the measured data and the theoretical predictions for the co- and cross-polarized backscattering coefficients of the dry concrete surface.

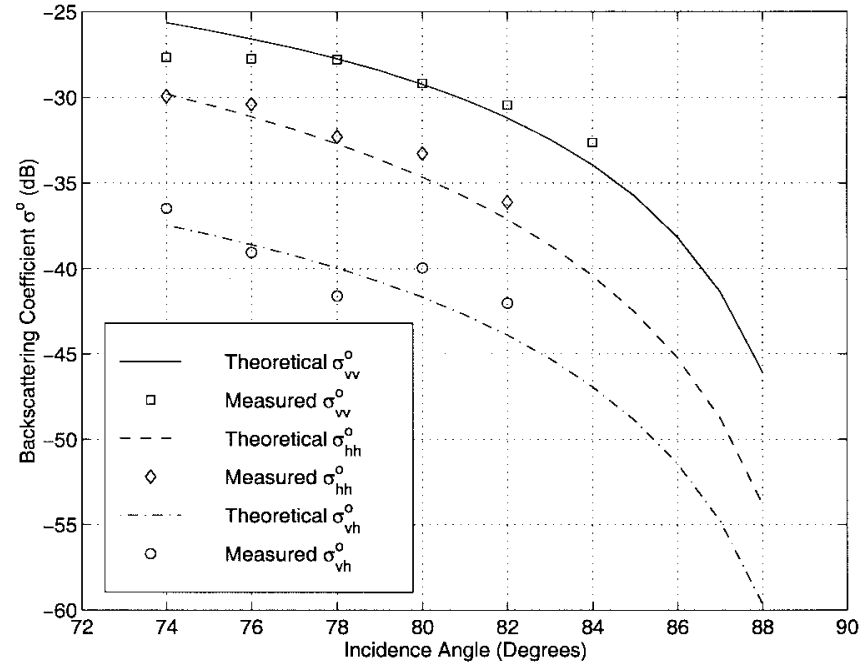

Fig. 6. The comparison between the measured data and the theoretdical predictions for the copolarized and cross-polarized backscattering coefficients of the ice-covered concrete surface.

permittivity fluctuation in concrete medium is much lower than that in asphalt medium. Fig. 5 shows the comparison between the theoretical values predicted by the model and the measured data for the dry concrete surfaces. Excellent agreement is observed. The ice-covered concrete surface is considered next. The dielectric constant of ice at $0^{\circ} \mathrm{C}$ (the temperature during the measurement) is chosen to be $\epsilon_{i}=3.15+j 0.27$ [5]. Fig. 6 displays an excellent agreement between the measured data and the values predicted by the model. The results of the phase difference statistics are illustrated in Figs. 7 and 8 . The discrepancy observed between the measured and

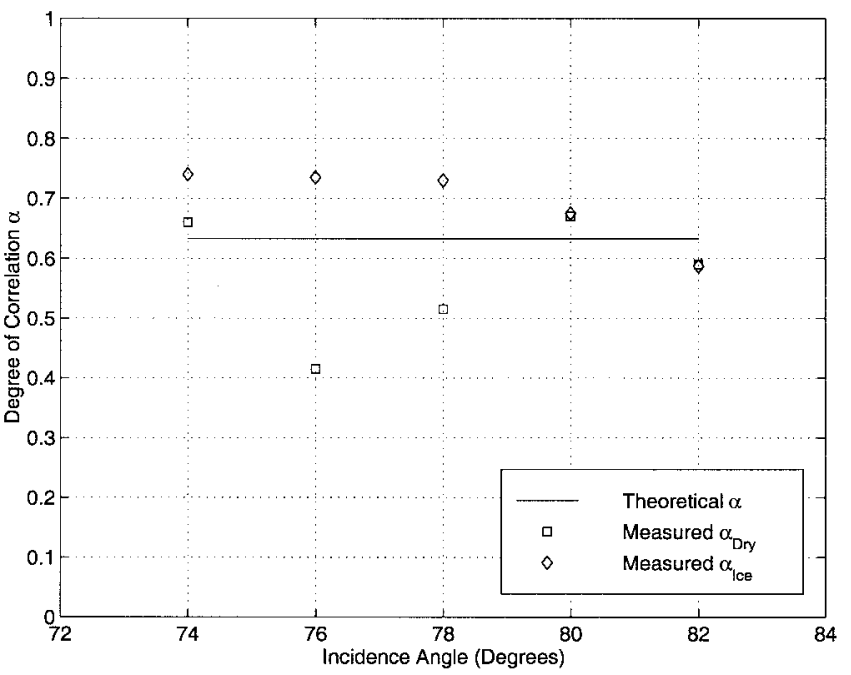

Fig. 7. The measured and calculated degree of correlation $(\alpha)$ of the dry and the ice-covered concrete surfaces.

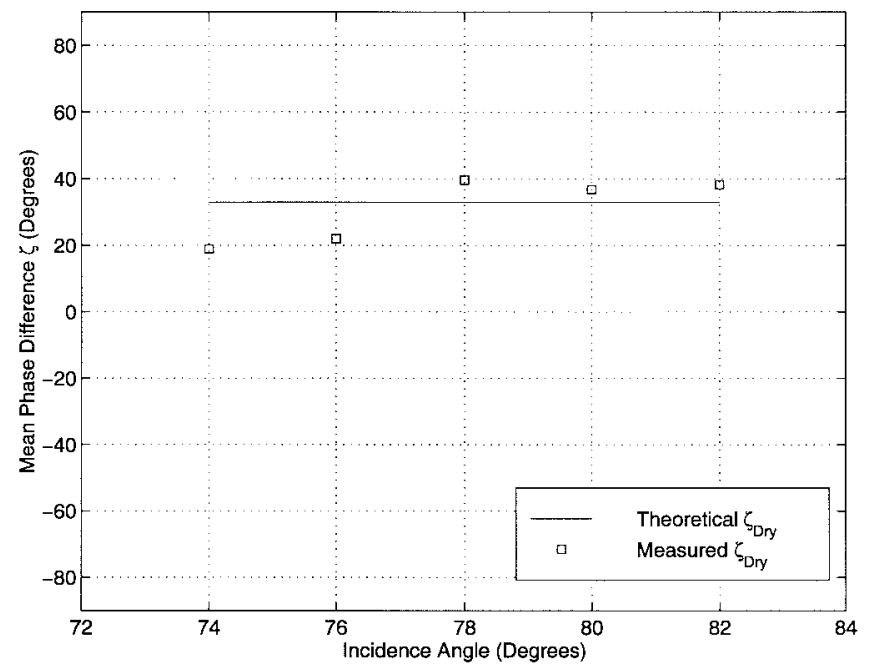

Fig. 8. The measured and calculated mean phase difference $(\zeta)$ of the dry asphalt surface.

TABLE III

The SCATtering COEFFicient $\kappa_{s, s}$, ABSORPTIONCOEFFICIENT $\kappa_{a, s}$, AND Extinction CoEfFicient $\kappa_{e, s}$ OF a SNOW Medium with Volume Fraction 0.0742 and Average Particle Radius $0.25 \mathrm{~mm}$ at $94 \mathrm{GHz}$

\begin{tabular}{c|c|c}
\hline$\kappa_{s, s}$ & $\kappa_{a, s}$ & $\kappa_{e, s}$ \\
\hline 5.7818 & 1.2574 & 7.0392 \\
\hline
\end{tabular}

TABLE IV

Normalized Elements of Phase Matrix of Asphalt Mixtures at $94 \mathrm{GHz}$ Obtained from the Polarimetric Backscatter Measurements

\begin{tabular}{c|c|c|c}
\hline$p_{1, a} / \kappa_{e, a}$ & $p_{2, a} / \kappa_{e, a}$ & $p_{3, a} / \kappa_{e, a}$ & $p_{4, a} / \kappa_{e, a}$ \\
\hline $2.36 \times 10^{-2}$ & $4.72 \times 10^{-3}$ & $1.16 \times 10^{-2}$ & $1.40 \times 10^{-3}$ \\
\hline
\end{tabular}

theoretical values of $\alpha$ can be attributed to the phase errors caused by the motion of the radar platform. For the mean phase difference $\zeta$, an average difference of less than $10^{\circ}$ is achieved.

The backscatter response of the snow-covered smooth asphalt surface is investigated next. The snow examined here is fresh dry snow with a thickness of $6.4 \mathrm{~mm}$ and a density 
of $0.068 \mathrm{~g} / \mathrm{cm}^{3}$. The average size of the ice crystals was measured to be $0.25 \mathrm{~mm}$ and the dielectric constant of the ice crystals of $\epsilon_{r}=3.15+j 0.025$ is used according to the ambient temperature of $-20^{\circ} \mathrm{C}$ during the measurement [5]. The scattering, absorption, and extinction coefficients of the snow medium are calculated from (16) and (17) and are listed in Table III. On the other hand, the extinction and phase matrices of the underlying asphalt layer were determined experimentally as described in [1]. Table IV lists the normalized elements of the phase matrix of the asphalt mixture at $94 \mathrm{GHz}$ which were reported in [1]. Fig. 9(a)-(c) compares the measured backscattering coefficients with the simulation results. The individual backscatter contributions from the snow layer and the asphalt layer are also included. It is quite obvious that $\sigma_{h h}^{\circ}$ is dominated by the backscatter from the snow layer. For vertical polarization, the volume scattering from snow and asphalt are comparable, whereas for cross-polarized term, backscatter from asphalt is the dominant component.

The last case investigated is the backscatter response of the rough asphalt surfaces. The site selected for the measurement has an rms height of $0.9 \mathrm{~mm}$ and a correlation length of $6.3 \mathrm{~mm}$. The correlation function is approximated by an exponential function. The overall backscatter response is computed from the superposition the volume scattering from the asphalt mixtures and the surface scattering from the rough interface. Since the rms slope of the surface is relatively low and the angular dependence of the volume scattering is very gentle, the volume scattering component is calculated using the hybrid scattering model assuming a smooth interface. The IEM is employed to calculate the surface scattering response using the surface roughness statistics. Fig. 10(a) and (b) compares the measured data to the theoretical predictions for the copolarized backscattering coefficients of the rough asphalt surface. The individual contributions from the volume and surface scattering components are also shown. The comparison demonstrates good agreement between the experimental data and the theoretical results. The IEM formulation for crosspolarized backscatter does not converge and hence is not compared. Fig. 11 shows the measured data and the volume scattering component of the cross-polarized backscatter response. It is shown that a significant portion of the crosspolarized backscatter is generated from the rough interface for which we do not have a reliable theoretical model. It is worth mentioning that an ice-covered rough asphalt surface would behave as an ice-covered smooth asphalt surface since the dielectric constant of ice and that of the asphalt mixture are almost the same.

\section{CONCLUSION}

Polarimetric backscatter behavior of concrete and asphalt road surfaces under variety of conditions is investigated thoroughly at $W$-band frequencies and accurate theoretical models capable of predicting the backscatter response near grazing incidence are developed. The surfaces considered are 1) dry and ice-covered smooth concrete surfaces, 2) a snow-covered smooth asphalt surface, and 3) a rough asphalt surface. It

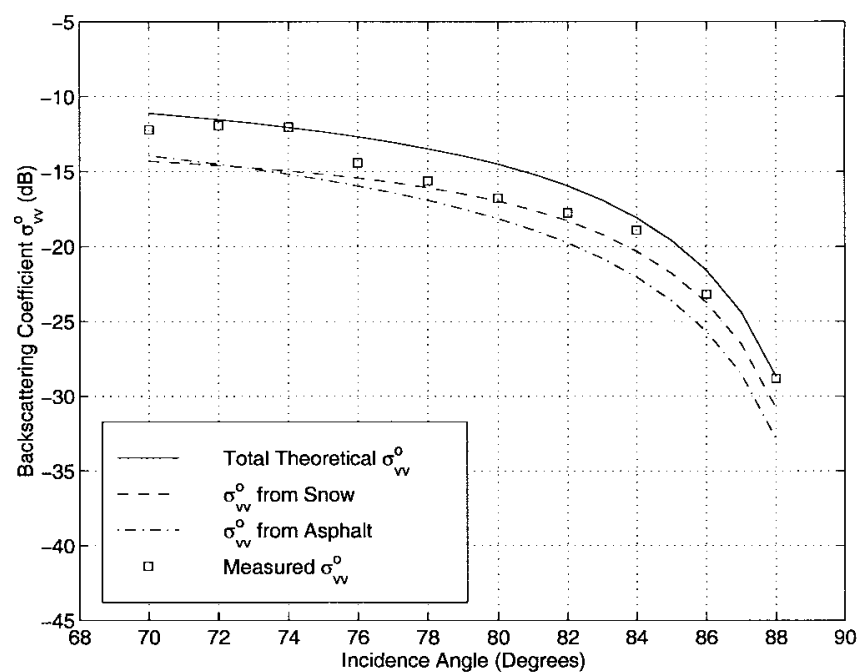

(a)

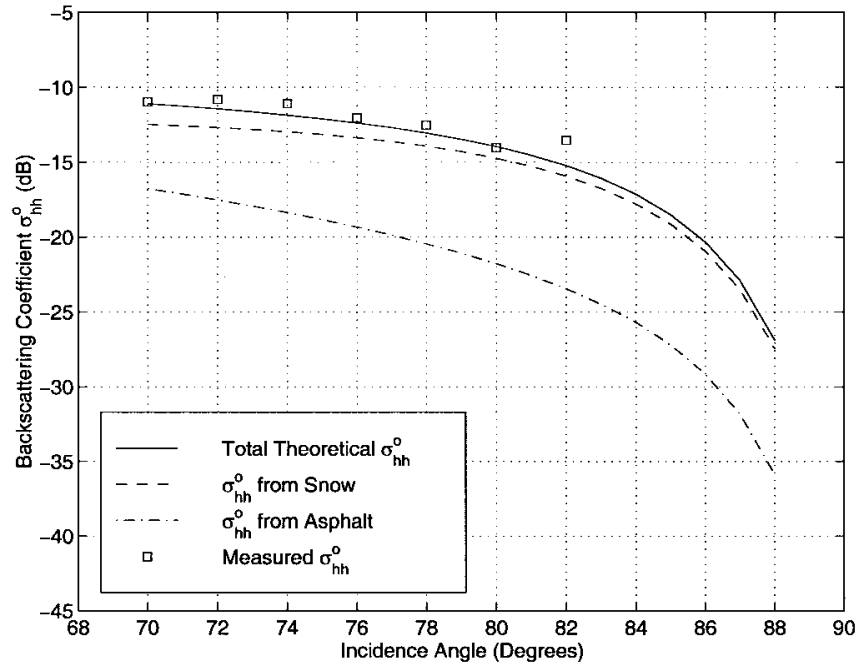

(b)

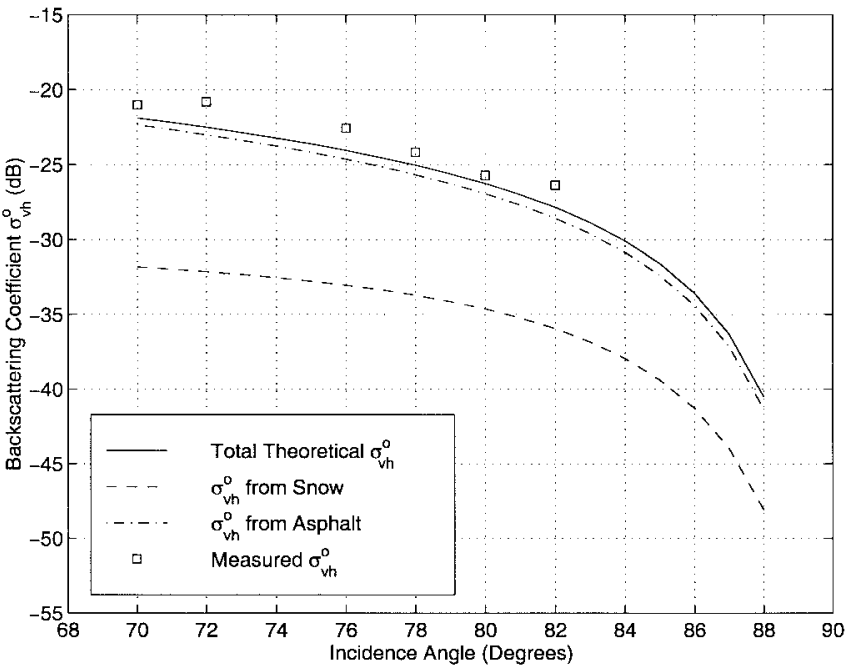

(c)

Fig. 9. The comparison between the measured data and the theoretical predictions for the copolarized and cross-polarized backscattering coefficients of the snow-covered smooth asphalt surface.

is shown that the hybrid volume scattering model developed earlier for smooth asphalt surfaces works well for concrete 


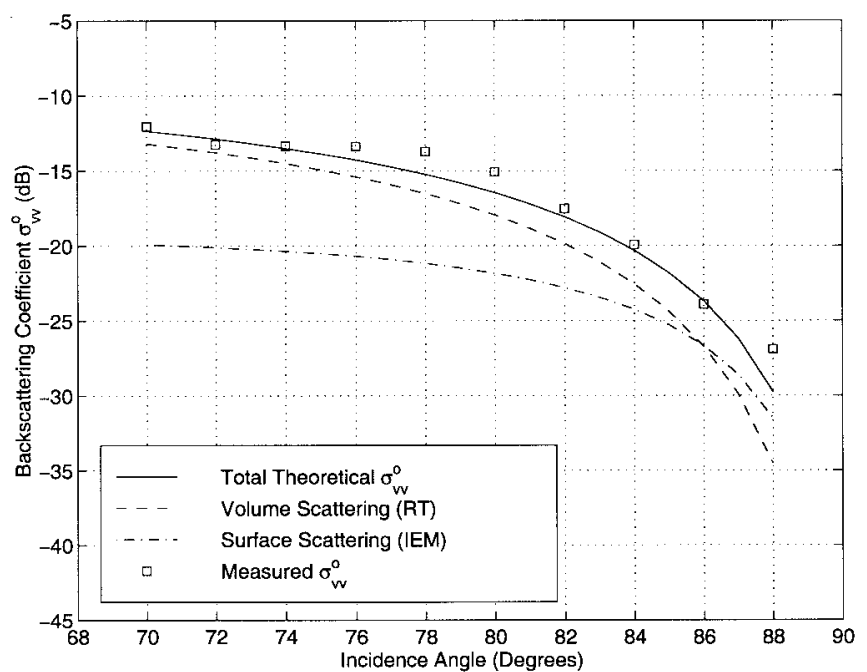

(a)

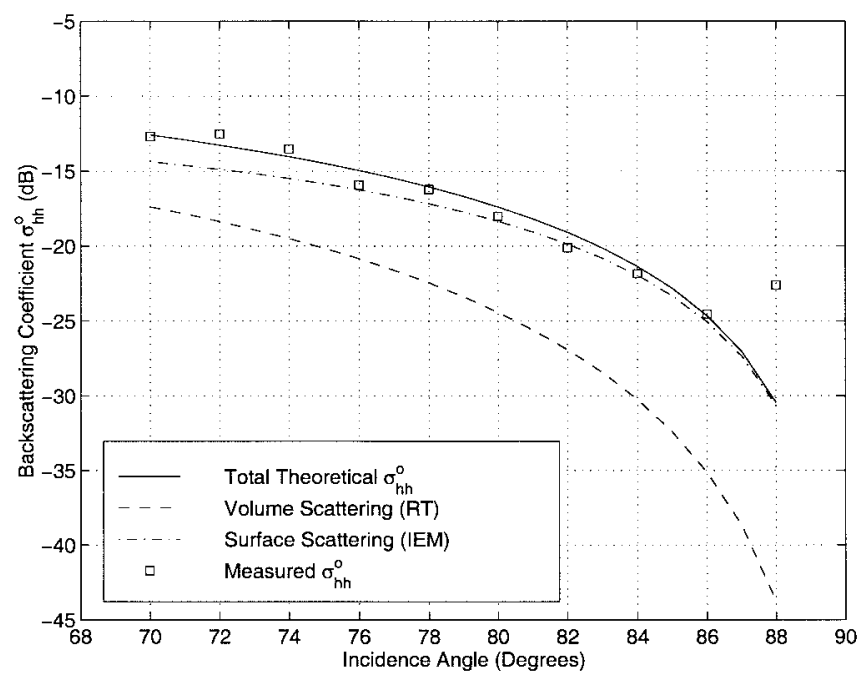

(b)

Fig. 10. The comparison between the measured data and the theoretical predictions for the copolarized backscattering coefficients of the rough asphalt surface.

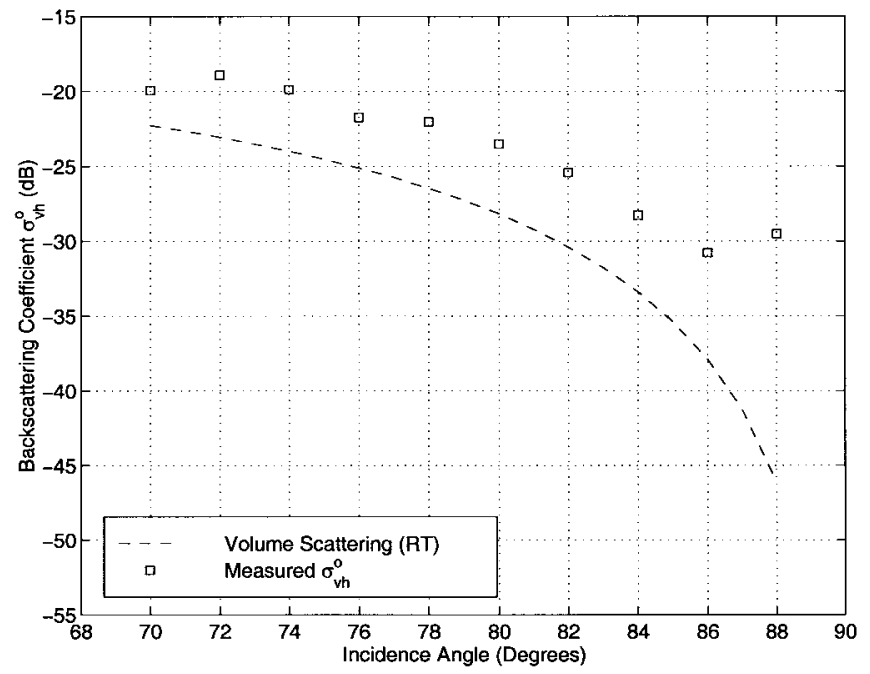

Fig. 11. The measured data and the volume scattering component of the cross-polarized backscattering coefficient of the rough asphalt surface (IEM formulation does not converge for the cross-polarized backscattering coefficient). surfaces once the normalized phase matrix elements $\left(\frac{p_{i, c}}{\kappa_{e}}\right)$ of the concrete medium were characterized experimentally. Excellent agreement is observed between the measured and predicted backscatter response of dry and ice-covered smooth concrete surfaces. For the snow-covered smooth asphalt surface, the first-order iterative solution of the radiative transfer equation is used to calculate the volume scattering from the snow layer for the copolarized components of the backscatter. To find the cross-polarized response, the second-order iterative solution is employed. The result is combined with the volume scattering from the underlying asphalt layer to predict the overall backscatter response. The volume scattering from asphalt and concrete mixtures depends on volume fraction and size of the constituent particles. Fortunately, asphalt and concrete mixtures are prepared according to a very welldefined procedure, hence, it is expected that experimentally characterized phase matrix elements and extinction coefficients be relatively constant. The scattering behavior from the rough asphalt surface includes both surface scattering from the rough interface and volume scattering from the asphalt mixture. The surface scattering can be predicted by the IEM formulation for the copolarized components and very good agreement between the theory and measurement is observed.

\section{REFERENCES}

[1] K. Sarabandi, E. S. Li, and A. Nashashibi, "Modeling and measurements of scattering from road surfaces at millimeter-wave frequencies," IEEE Trans. Antennas Propagat., vol. 45, pp. 1679-1688, Nov. 1997.

[2] M. Lax, "Multiple scattering of waves, II. The effective field in dense systems," Phys. Rev., vol. 85, no. 4, pp. 621-629, Feb. 15, 1952.

[3] L. Tsang, J. Kong, and R. Shin, Theory of Microwave Remote Sensing. New York: Wiley, 1985

[4] K. Sarabandi and E. S. Li, "A microstrip ring resonator for soil moisture measurements," IEEE Trans. Geosci. Remote Sensing, vol. 35, pp. 1223-1231, Sept. 1997.

[5] F. T. Ulaby, R. K. Moore, and A. K. Fung, Microwave Remote Sensing, vol. III. Norwood, MA: Artech House, ch. 13, and Appendices E2-E3, pp. $2020-2028$

[6] _ Microwave Remote Sensing, vol. II. Norwood, MA: Artech House, ch. 12, pp. 822-825.

[7] K. Sarabandi, "Electromagnetic scattering from vegetation canopies," Ph.D. dissertation, The University of Michigan, Ann Arbor, 1989.

[8] S. O. Rice, "Reflection of electromagnetic wave by slightly rough surfaces," Commun. Pure Appl. Math., vol. 4, pp. 351-378, 1951.

[9] M. Nieto-Vesperinas, "Depolarization of electromagnetic waves scattered from slightly rough random surfaces: A study by means of the extinction theorem," J. Opt. Soc., vol. 72, no. 5, pp. 539-547, 1982.

[10] P. Beckmann and A. Spizzichino, The Scattering of Electromagnetic Waves from Rough Surfaces. New York: Pergamon, 1963.

[11] D. Winebrenner and A. Ishimaru, "Investigation of a surface field phase perturbation technique for scattering from rough surfaces," Radio Sci. vol. 20, no. 2, pp. 161-170, Mar., Apr. 1985.

[12] E. Rodriguez and Y. Kim, "A unified perturbation expansion for surface scattering," Radio Sci., vol. 27, no. 1, pp. 79-93, Jan., Feb. 1992.

[13] K. Sarabandi, "Derivation of phase statistics of distributed targets from the Mueller matrix," Radio Sci., vol. 27, no. 5, pp. 553-560, 1992.

[14] R. Shin and J. A. Kong "Radiative transfer theory for active remote sensing of a homogeneous layer containing spherical scatterers," J. Appl. Phys., vol. 52, no. 6, pp. 4221-4230, June 1981.

[15] A. K. Fung, Microwave Scattering and Emission Models and Their Applications. Norwood, MA: Artech House, 1994, ch. 5.

[16] K. Sarabandi, " $\Delta k$-radar equivalent of interferometric SAR's: A theoretical study for determination of vegetation height," IEEE Trans. Geosci. Remote Sensing., vol. 35, pp. 1267-1276, Sept. 1997.

[17] A. Nashashibi, K. Sarabandi, and F. T. Ulaby, "A calibration technique for polarimetric coherent-on-receive radar system," IEEE Trans. Antennas Propagat. vol. 43, pp. 396-404, Apr. 1995. 


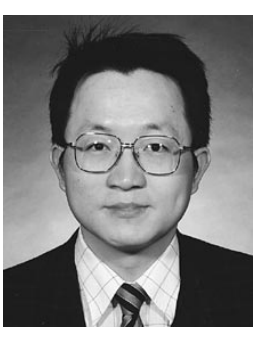

Eric S. Li (M'90-S'93-M'98) received the B.S. degree from the Tamkang University, Taipei, Taiwan, in 1986 and the M.S. degree in electrical engineering from the State University of New York, Stony Brook, in 1987. He entered the graduate program at the University of Michigan, Ann Arbor, in 1993 and received the Ph.D. degree in electrical engineering in 1998 .

From 1988 to 1992 he worked as a microwave engineer in the cellular phone industry. He is currently a Senior Research Engineer at EMAG Technologies, Inc., Ann Arbor. His interests include microwave circuits, millimeter-wave polarimetric radar systems, calibration and measurement techniques, and electromagnetic scattering.

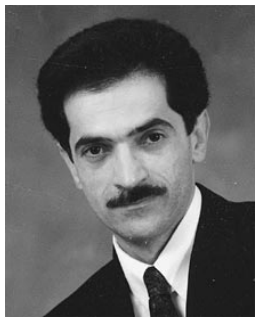

Kamal Sarabandi (S'87-M'90-SM'92) received the B.S. degree in electrical engineering from Sharif University of Technology, Tehran, Iran, in 1980. He entered the graduate program at the University of Michigan, Ann Arbor, in 1984 and received the M.S.E. degree in electrical engineering in 1986, and the M.S. degree in mathematics and the Ph.D. degree in electrical engineering in 1989.

From 1980 to 1984 he worked as a microwave engineer in the Telecommunication Research Center. He is presently an Associate Professor in the Department of Electrical Engineering and Computer Science at the University of Michigan. He has 18 years of experience with microwave sensors and radar systems. In the past eight years he has served as the Principal Investigator and Co-Investigator on many projects sponsored by NASA, JPL, ARO, ONR, ARL, and GM, all related in one way or another to microwave- and millimeterwave radar remote sensing. He has published many book chapters and more than 75 papers in refereed journals on electromagnetic scattering, random media modeling, microwave measurement techniques, radar calibration, application of neural networks in inverse scattering problems, and microwave sensors. He has also had more than 130 papers and invited presentations in national and international conferences and symposia on similar subjects.

Dr. Sarabandi is listed in American Men \& Women of Science and Who's Who in Electromagnetics. He has been a member of the IEEE Geoscience and Remote Sensing ADCOM since January of 1998 and served as the Chairman of Geoscience and Remote Sensing Society Southeastern Michigan chapter from 1992 to 1998. He is also a member of Commission F of URSI and of The Electromagnetic Academy. He was a recipient of a 1996 Teaching Excellence Award, the 1997 Henry Russel Award from the Regent of The University of Michigan, and the 1999 GAAC Distinguished Lecturer Award from the German Federal Ministry for Education, Science, and Technology. 